Revue belge de géographie

\title{
Les systèmes de gouvernance des territoires transfrontaliers : la mise en œuvre du règlement européen sur les GECT (Groupements Européens de Coopération Territoriale)
}

The implementation of the EU regulation on EGCTs (European Groupings for Territorial Cooperation) in crossborder territory governance systems

\section{Valérie Biot}

\section{(2) OpenEdition Journals}

\section{Édition électronique}

URL : http://journals.openedition.org/belgeo/10565

DOI : 10.4000/belgeo.10565

ISSN : 2294-9135

\section{Éditeur :}

National Committee of Geography of Belgium, Société Royale Belge de Géographie

\section{Référence électronique}

Valérie Biot, «Les systèmes de gouvernance des territoires transfrontaliers : la mise en œuvre du règlement européen sur les GECT (Groupements Européens de Coopération Territoriale) », Belgeo [En ligne], 1 | 2013, mis en ligne le 31 octobre 2013, consulté le 19 avril 2019. URL : http:// journals.openedition.org/belgeo/10565; DOI : 10.4000/belgeo.10565

Ce document a été généré automatiquement le 19 avril 2019

Belgeo est mis à disposition selon les termes de la licence Creative Commons Attribution 4.0 International. 


\section{Les systèmes de gouvernance des territoires transfrontaliers : la mise en œuvre du règlement européen sur les GECT (Groupements Européens de Coopération Territoriale)}

The implementation of the EU regulation on EGCTs (European Groupings for Territorial Cooperation) in crossborder territory governance systems

Valérie Biot

\section{Introduction : pourquoi une approche par la gouvernance}

1 La question de la gouvernance est un défi permanent pour l'Union européenne. En 2001, un Livre Blanc sur le sujet était publié par la Commission (COM (2001)428 final). Depuis l'inclusion dans le Traité européen de Lisbonne de la cohésion territoriale comme un objectif de l'Union européenne, la gouvernance territoriale s'est également vu reconnaître une place à part entière (rapport Barca, 2009).

\section{Gouvernance et gouvernance territoriale}

Le concept de gouvernance englobe, dans notre acception, les processus verticaux et horizontaux d'interaction et de négociation entre acteurs publics, mais également entre acteurs publics et privés. Le concept a par exemple été développé en ce sens dans plusieurs travaux sur la "gouvernance urbaine" (Jouve, 2003). Il est parfois précisé que la gouvernance est "multiniveaux", à savoir incluant plusieurs niveaux de pouvoirs publics 
(Hooghe \& Marks, 2001) et/ou "multicanaux" (ESPON 2.3.2, 2006), ce qui recouvre la diversité des types d'acteurs. Cette gouvernance, comprise comme une nouvelle modalité d'exercice du pouvoir, peut s'exercer dans un secteur particulier ou sur un territoire donné, ou encore à l'échelle européenne tout entière, puisque l'Union européenne utilise ce concept et ses déclinaisons dans un but à la fois fonctionnel - comment faire fonctionner ce "système" novateur ${ }^{1}$ - et de légitimation par différentes voies (Levrat \& Esposito, 2010).

3 La gouvernance territoriale quant à elle peut s'analyser selon deux interprétations : d'une part elle recouvre la dimension territoriale de tout processus de gouvernance (ESPON TANGO, 2013), d'autre part, elle correspond à la gouvernance d'un territoire spécifique, par exemple métropolitain ou transfrontalier. C'est cette dernière interprétation qui nous intéresse ici, car la particularité des territoires transfrontaliers est double : d'une part, comme d'autres territoires, par exemple métropolitains, ils concentrent un enchevêtrement d'autorités et de compétences diverses, d'autre part, plus spécifiquement, ils sont considérés comme des "laboratoires" de l'intégration européenne, ou du moins de son "vivre ensemble", et concernent, au moins, deux Etats souverains (Gorzelak et Zawalinska, 2013).

\section{Gouvernance transfrontalière}

4 La gouvernance des espaces transfrontaliers concentre de nombreux enjeux de la gouvernance européenne.

5 En effet, sur ces "espaces", concept très large et sans concrétisation politique, les autorités publiques de certains "territoires" infranationaux - concrétisés par des limites administratives et/ou politiques, ainsi que par des référents fonctionnels et/ou morphologiques - s'organisent dans le but de collaborer sur un territoire à la fois infranational et "international', ressortissant à au moins deux Etats souverains.

6 Les enjeux autour de ces nouvelles créations politiques dépassent souvent largement les raisons pratiques et/ou stratégiques qui ont localement entraîné l'émergence de cette collaboration, puisqu'ils interrogent la souveraineté des Etats en promouvant une plus forte intégration européenne, mais aussi en permettant l'émergence de nouvelles entités politiques.

7 La gouvernance de ces territoires transfrontaliers représente également un enjeu en matière de recherche sur la gouvernance en général, car elle doit tenir compte des multiples acteurs en présence, de leurs influences diverses et de leurs intérêts multiples, sinon contradictoires.

8 Ces acteurs sont d'une part des acteurs publics investis d'autorité sur le territoire concerné : des Etats souverains, mais également des pouvoirs délégués à de nombreux niveaux infranationaux - régions, communes, provinces, institutions supramunicipales..., ce qui correspond au concept de gouvernance multiniveaux (multilevel).

D'autre part, outre des acteurs publics investis d'une autorité directe, de nombreux autres types d'acteurs entrent en jeu : soit publics sans autorité directe sur le territoire donné, soit non publics, à savoir les citoyens, organisés ou non en "société civile", et enfin les acteurs économiques, ce que recouvre le concept de gouvernance multicanaux ( multichannel). La particularité des modes de gouvernance de ces territoires 
transfrontaliers, leur essence même, est de devoir fonctionner sur base du consensus et de la coopération.

10 A travers le programme INTERREG, l'Union européenne a fortement soutenu les initiatives transfrontalières et transrégionales et continue à le faire par le biais de nouvelles initiatives en termes de gouvernance et d'organisation stratégique et pratique.

11 Deux initiatives en particulier sont intéressantes comme modèle possible pour une organisation novatrice et modulable de la gouvernance, adaptée à des objectifs et à des territoires divers: les stratégies macrorégionales et les Groupements Européens de Coopération Territoriale, les GECT.

12 Les stratégies macrorégionales - à ce jour la stratégie Baltique et la stratégie Danube s'étendent sur de vastes espaces, plusieurs pays, et ne font pas l'objet d'une régulation particulière de l'UE, qui les soutient cependant activement (COM (2011) 610 final/2, 2011/0273 COD). Nous ne les traiterons pas ici².

13 Le GECT est quant à lui un nouvel instrument juridique européen, institué par un règlement adopté en 2006 par la Commission et le Parlement européens en vue d'organiser la coopération territoriale sur des bases harmonisées, flexibles mais stables, et européennes (EC 1082/2006).

14 L'objectif de cet article est d'étudier sa mise en œuvre et ses implications en termes de gouvernance, d'une part en identifiant sa pertinence en termes d'analyse des systèmes de gouvernance, d'autre part en proposant une grille de lecture comparative des GECT fondée sur les principales caractéristiques des systèmes utilisés par les GECT existants.

\section{Un règlement européen directement applicable dans les Etats membres de l'UE : quelle concrétisation?}

15 Le GECT est formalisé par le règlement 1082/2006 du Parlement Européen et du Conseil et est entré en vigueur le $1^{\text {er }}$ août 2006. Il vise à permettre à des entités publiques de différents Etats membres (États membres) de l'Union européenne de se réunir en une nouvelle entité dotée de la personnalité juridique. Les membres d'un GECT peuvent être des Etats, des autorités locales, régionales, ou toute autre autorité publique.

Contrairement au caractère de droit international de la coopération territoriale entre deux Etats souverains, le GECT est gouverné par un règlement européen, ce qui signifie qu'il revêt une portée générale, est obligatoire dans tous ses éléments et est directement applicable dans tous les États membres, à la différence d'une directive, qui doit être transposée en droit national ${ }^{3}$. Le cadre légal d'un GECT provient donc du règlement européen (EC) 108/2006, des dispositions contraignantes établies par les membres du GECT (Convention et Statut, cf. infra), et de la loi de l'Etat membre dans lequel le GECT est enregistré. Cas particulier, il est cependant précisé que ce règlement doit être complété par des "dispositions nationales", ce qui entraine certaines incertitudes (cf. infra).

\section{Des éléments obligatoires, une grande flexibilité}

Le règlement GECT énonce certains éléments obligatoires pour tout GECT, mais laisse ensuite à ses membres une grande possibilité d'adaptation. 

Une Convention doit en préciser le nom, le territoire, les objectifs et les tâches; en lien avec la Convention, des Statuts doivent être adoptés, précisant le mode de fonctionnement des institutions du GECT et leurs compétences, la procédure de prise de décision, les langues de fonctionnement et les contributions financières. Ces deux documents sont cruciaux, et le fait que les membres du GETC puissent parvenir à un accord sur ces différents points est un pas décisif dans la formalisation d'une gouvernance stable et dans le processus de prise de décision politique. Tous les membres du GECT sont impliqués dans l'établissement et l'approbation d'orientations stratégiques et de l'architecture institutionnelle du GECT. charge des tâches clés comme l'établissement du budget; et un directeur, qui représente le GECT et agit en son nom. Le GECT peut décider d'instituer d'autres organes, par exemple un forum de la société civile, un bureau restreint à certains membres, des groupes de travail. Il peut également inviter dans des organes ouverts ad hoc des représentants d'autres coopérations pour des objectifs ou des thématiques spécifiques. Ce dispositif permet une grande faculté d'adaptation à la fois spatiale et temporelle.

Les GECT doivent avoir pour vocation la coopération territoriale, que ce soit de manière ciblée ou généraliste, ou encore en prenant en charge un programme de l'UE (Interreg). Les ressources financières utilisées ne doivent toutefois pas nécessairement provenir de l'UE.

21 Par ailleurs, si les membres d'un GECT doivent être des autorités publiques appartenant à au moins deux Etats membres ${ }^{4}$, aucun niveau d'autorité particulier n'est requis et plusieurs niveaux d'autorité peuvent se retrouver membres. Cette possibilité de gouvernance multiniveaux est une valeur ajoutée importante de cet instrument, un Etat pouvant dans ce cadre collaborer directement avec des entités infranationales. Dans les faits cependant, très peu de GECT sont "multiniveaux", la plupart regroupant des partenaires de même niveau d'autorité dans leurs pays respectifs, le plus souvent au niveau local, de sorte que l'assemblée du GECT peut rassembler plus d'une centaine de représentants (maximum à ce jour, 170 communes hongroises et slovaques).

Enfin, le fait que les Etats membres aient mis en œuvre cette réglementation en y insérant des "dispositions nationales", ajoute encore à la diversité des systèmes de gouvernance mis en place. En effet, les Etats peuvent par exemple décider d'accepter ou non la coopération avec des Etats non membres, ou si le GECT peut avoir un domaine d'action limité ou illimité, ou encore, ce qui est particulièrement surprenant, si le droit applicable est privé ou public.

\section{Mise en œuvre lente et différenciée}

Ce règlement a été fortement soutenu par le Comité des Régions (CDR, 2006, 2008, 2010, 2011, 2012), ainsi que par la Direction générale de la Commission européenne en charge de la coopération territoriale. Néanmoins, les débuts de son utilisation ont été lents, et très variables selon les pays.

En janvier 2013, trente GECT sont formalisés et installés, ce qui est loin de couvrir le nombre de coopérations territoriales existantes, que ce soit dans le cadre d'Interreg ou d'autres programmes. 
Des GECT sont présents dans 15 Etats membres de l'UE, en France (12), Hongrie (11), Espagne (9), Slovaquie (8), Belgique (4), Italie (4), Allemagne (3), Portugal (3), Chypre (2), Roumanie (2), Slovénie (2), Autriche (1), Grèce (1), Luxembourg (1), Pays-Bas (1), et dans un pays voisin non membre de l'UE, la Serbie (1). Ils sont totalement absents des pays du Nord de l'UE : Scandinavie, Grande-Bretagne, Irlande, Pays Baltes. Ils sont par contre bien utilisés en Europe centrale, à l'exception de la Pologne et de la République tchèque. La plus grande concentration de GECT se retrouve entre la France et ses voisins (Belgique, Espagne, Allemagne), entre le Portugal et l'Espagne, la Hongrie et ses pays voisins, en particulier la Slovaquie. Il n'existait début 2013 qu'un seul GECT entre un ancien et un nouvel Etat membre (Italie, Slovénie, 2011).

Figure 1. Implantation des GECT en été 2013 (www.cesci-net.eu).

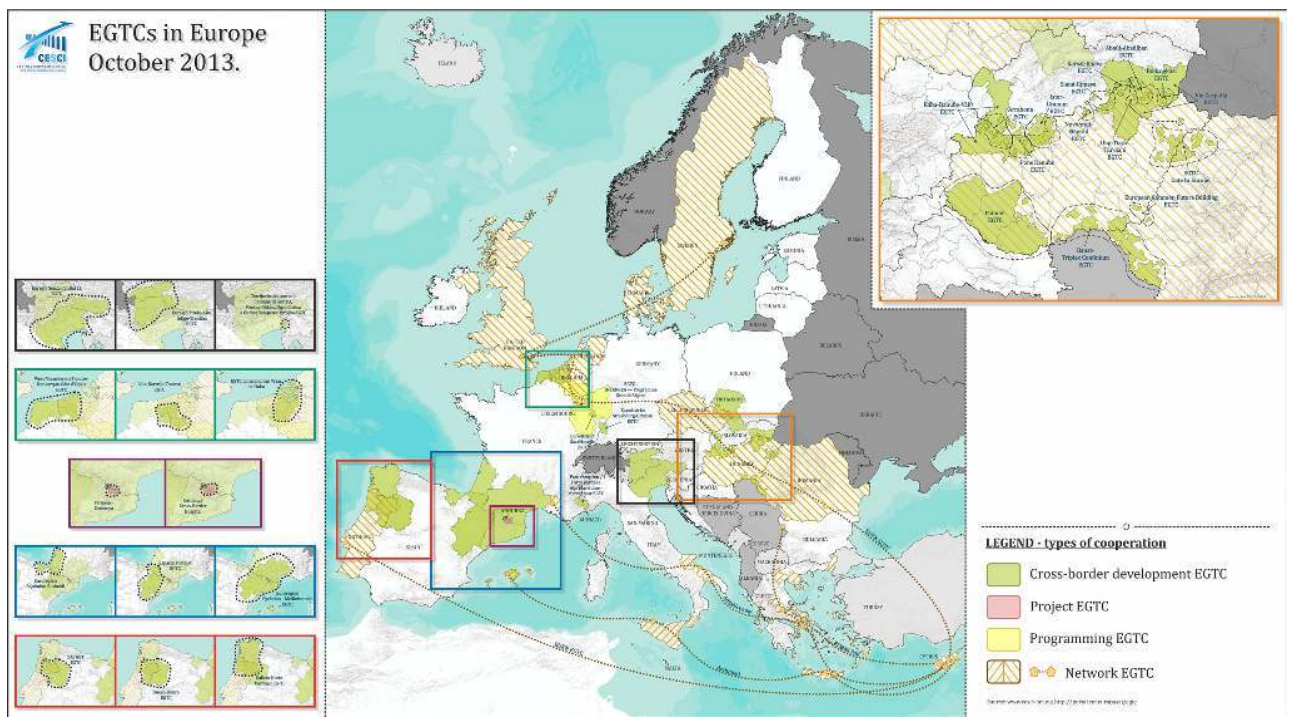

\section{Raisons possibles et évolutions futures}

Plusieurs raisons expliquent cette lenteur, et cette différence dans la mise en œuvre. D'une part, le règlement, adopté en 2006, intervenait trop tard pour être utilisé lors de la programmation 2007-2013 (un seul GECT est un organe de gestion d'un programme Interreg : la Grande Région). D'autre part, certains pays ont déjà développé des structures de coopération dont ils s'estiment satisfaits (en particulier autour de la Baltique).

Par ailleurs, plusieurs problèmes d'ordre politique, administratif ou technique ont freiné l'utilisation de ce nouvel outil. Ils sont listés et développés dans le rapport de la Commission sur l'application du règlement sur les GECT (COM (2011) 462 final) ${ }^{5}$. En résumé, le règlement est loin d'apporter une solution aux problèmes quotidiens d'une coopération transfrontalière, et dans certains cas il introduit des difficultés nouvelles (par exemple le statut du staff employé par les GECT) ; la distinction entre la Convention et les Statuts n'est pas claire ; l'instrument est nouveau et doit être testé ; il ne permet pas de créer un GECT bilatéral avec un Etat non membre; il est encore peu reconnu par les services mêmes de la Commission autres que la DG Regio.

Enfin, deux écueils concernent directement la façon dont les Etats membres accueillent ce nouvel instrument: d'une part les Etats peuvent compléter le règlement par des "dispositions nationales", ce qui a entraîné d'importantes différences dans la mise en 
oeuvre des GECT, et il se trouve en contradiction avec l'objectif recherché d'harmonisation et de simplification des règles et législations applicables. D'autre part, une fois le règlement effectivement mis en œuvre, un GECT souhaitant s'établir doit se soumettre à l'approbation des États membres auxquels ses membres ressortissent, un aspect juridique quelque peu ambigu si l'on se réfère à l'essence du règlement, qui est de s'appliquer directement, sans nécessiter la transposition en droit national.

La Commission a déposé fin 2011 une proposition de révision du règlement (COM 2011610 final 2011/0272 COD) afin de tenter de répondre à ces failles. Plusieurs solutions sont apportées, et la proposition a reçu un bon accueil du Comité des Régions (2012/C 113/06). Néanmoins, la volonté d'harmonisation bute toujours sur la souveraineté des États membres, et les "dispositions nationales" différenciées, ainsi que sur les procédures d'approbation par les États membres des GECT mis sur pied ${ }^{6}$.

\section{Le GECT : quelle utilité comme instrument d'analyse ? Propositions pour une classification des systèmes de gouvernance transfrontalière}

\section{Quelle utilité comme instrument d'analyse?}

L'analyse des coopérations transfrontalières par leurs systèmes de gouvernance concerne directement la concrétisation en termes politiques d'éléments fonctionnels (un bassin d'emploi par exemple) et/ou morphologiques (en particulier une urbanisation continue), ou encore géographiques (le partage d'un bassin fluvial, d'une zone naturelle...). L'approche par la gouvernance prend en compte également certains types de coopérations transfrontalières qui utilisent le territoire transfrontalier afin d'atteindre un seuil requis permettant des projets de plus large envergure, et/ou permettant de s'inscrire dans un cadre européen. On parlera donc plutôt dans un cas de géographie "fonctionnelle", imposée par des faits concrets, dans l'autre de coopération stratégique, émanant d'une volonté politique.

31 Cette approche permet une investigation à travers des éléments clés pour l'existence d'une coopération: qui est à l'initiative de cette coopération, quels acteurs ont été mobilisés, quelles sont les "forces motrices", quels types d'acteurs sont impliqués, quel partenariat est mis en place, quelles sont les motivations pour formaliser cette coopération, quelle est la part de "bottom up" et de "top down" dans la conduite de cette coopération? Sur le plan pratique, quel type de structure est mis en place, avec quel potentiel de délégation et d'autonomie, et sur quel territoire?

L'utilisation des GECT comme cas d'étude permet une identification de coopérations ayant opté pour un certain degré de formalisation, et possédant un cadre contraignant identique: une convention et des statuts, une assemblée et un directeur, un objectif général de coopération territoriale. Ce cadre peut ensuite se décliner de façons très diverses, permettant une grande faculté d'adaptation à des contextes multiples dans un cadre stable. Cette grande diversité a cependant son revers, surtout lié aux modalités nationales de mise en œuvre du règlement: aucune harmonisation ne se réalise, et il règne une insécurité juridique préjudiciable à la pérennisation des coopérations transfrontalières. 
L'analyse de cette diversité est fondamentale pour imaginer des systèmes de gouvernance efficaces, si l'on s'inscrit dans le paradigme défendu par le rapport Barca (Barca, 2009) "one size does not fit all" : il n'existe pas une recette convenant à tous et toutes, les solutions doivent tenir compte du contexte et de l'échelle géographique, ainsi que du stade d'évolution des coopérations.

\section{Tentative de classification des différents systèmes mis en place}

Dans le cadre d'un projet de recherche européen (ESPON TERCO, 2012) nous avons établi un tableau illustrant la diversité des systèmes de gouvernance à l'œuvre dans les GECT existant à la fin 2011.

Nous avons utilisé d'une part une recherche documentaire ${ }^{7}$ sur les 24 GECT existant fin $2011^{8}$; d'autre part trois cas d'études approfondis, réalisés par des experts des territoires en question ${ }^{9}$ : il s'agissait de l'Eurométropole Lille-Courtrai-Tournai (LIKOTO), le premier GECT implanté, et un des meilleurs exemples de multigouvernance réelle et de l'élaboration volontariste d'une stratégie de construction d'une Eurométropole; le GECT Grande Région, seul GECT organe de gestion d'un programme Interreg; et un cas d'étude dans la macrorégion Danube, afin de tenter d'identifier d'éventuels liens entre GECT et stratégies macrorégionales (liens qui s'avèrent peu probants à l'heure actuelle).

Pour tenter une classification par les systèmes de gouvernance en place dans le cadre des GECT, plusieurs entrées sont possibles. Les GECT implantés mêlent à loisir ces différentes clés d'entrée, car il n'existe pas de corrélation entre elles. Le choix devra donc se faire en fonction du questionnement de la recherche.

\section{Clés d'entrée pour une classification des systèmes de gouvernance transfrontaliers}

\begin{tabular}{|l|l|}
\hline Type de coopération & $\begin{array}{l}\text { Tous GECT existants sont établis en vue d'une coopération } \\
\text { transfrontalière impliquant au moins deux Etats membres de l'Union } \\
\text { européenne. } \\
\text { Certains couvrent cependant des territoires très vastes, ou encore } \\
\text { impliquent plus de deux Etats membres. } \\
\text { Un seul GECT (trilatéral) implique également un Etat non membre de } \\
\text { l'UE. } \\
\text { Dans tous ces cas, il existe néanmoins une proximité et continuité } \\
\text { géographique. } \\
\text { Seules exceptions à ce caractère transfrontalier du GECT, 2 réseaux } \\
\text { thématiques sans proximité géographique: } \\
\text { - l'un entre des Iles de la Méditerranée (Archiméd); } \\
\text { - l'autre entre des communes présentes sur le continent autour de la } \\
\text { Méditerranée (Amphictiony). }\end{array}$ \\
\hline
\end{tabular}




\begin{tabular}{|c|c|}
\hline Objectif & $\begin{array}{l}\text { La plupart des GECT en exercice s'occupent de coopération sur un large } \\
\text { éventail de matières - environnement, sécurité, transports, culture, } \\
\text { implantation d'entreprises... - tentant une gestion intégrée d'un } \\
\text { territoire complexe. } \\
\text { Certains sont cependant destinés à piloter un projet de coopération } \\
\text { territoriale spécifique, par exemple un hôpital transfrontalier, ou un } \\
\text { parc naturel transfrontalier. } \\
\text { Un seul GECT à ce jour est un organe de gestion d'un programme } \\
\text { européen Interreg. }\end{array}$ \\
\hline Loi & $\begin{array}{l}\text { Des GECT ont été établis dans } 15 \text { États membres. } \\
\text { Deux États membres ont décidé de mettre en œuvre le règlement GECT } \\
\text { sous un régime de droit privé (GR, SK), malgré le fait que seules des } \\
\text { autorités publiques peuvent être membres d'un GECT. }\end{array}$ \\
\hline Compétence & $\begin{array}{l}\text { Aucun GECT ne dispose de compétences déléguées. Ils sont en charge de } \\
\text { "missions" et de la mise en oeuvre de projets (transfrontaliers). } \\
\text { L'évolution en la matière sera un aspect clé de l'analyse en termes de } \\
\text { gouvernance. A l'heure actuelle par exemple, quelques GECT mettent en } \\
\text { oeuvre un processus d'élaboration d'une stratégie globale sur leur } \\
\text { territoire. }\end{array}$ \\
\hline Structure commune & $\begin{array}{l}\text { Deux structures communes de gestion du GECT ont pu être clairement } \\
\text { identifiées (GECT LIKOTO et Grande Région), avec un bureau, du } \\
\text { personnel, un budget de fonctionnement pour le GECT lui même. } \\
\text { Dans la grande majorité des GECT, ce sont les structures locales qui } \\
\text { prennent en charge le suivi du GECT, avec ou sans financement } \\
\text { spécifique. } \\
\text { L'existence ou non d'une structure commune ne préjuge pas } \\
\text { nécessairement du stade d'évolution de la coopération, ni de son } \\
\text { envergure, mais le manque de ressources, en temps et en financement, } \\
\text { handicape l'évolution future du GECT. }\end{array}$ \\
\hline Organes additionnels & $\begin{array}{l}\text { La possibilité d'instaurer des organes additionnels associés au GECT est } \\
\text { un atout majeur du règlement GECT. } \\
\text { Plusieurs GECT ont institué un Bureau permettant un suivi plus assidu } \\
\text { des travaux que l'Assemblée annuelle des membres. } \\
\text { Des entités ad hoc sont aussi créées pour permettre la coopération avec } \\
\text { d'autres partenaires, y compris hors EU (par exemple la Turquie dans le } \\
\text { cas d'Amphictiony). } \\
\text { La société civile se voit parfois (très peu jusqu'à présent) impliquée par } \\
\text { le biais de "Forum". }\end{array}$ \\
\hline $\begin{array}{l}\text { Types d'acteurs } \\
\text { impliqués } \\
\text { (multicanaux) }\end{array}$ & $\begin{array}{l}\text { Le GECT n'est ouvert qu'aux autorités publiques, dès lors les acteurs } \\
\text { impliqués de façon institutionnelle sont tous publics (cf. règlement } \\
\text { GECT }^{10} \text { ). } \\
\text { Néanmoins, la possibilité d'ajouter des organes additionnels facultatifs } \\
\text { aux organes requis par le Règlement GECT (un directeur et une } \\
\text { assemblée des membres) permet d'impliquer de façons différenciées } \\
\text { d'autres types d'acteurs, non publics, une possibilité encore peu utilisée } \\
\text { jusqu'à présent. }\end{array}$ \\
\hline
\end{tabular}




\begin{tabular}{|l|l|}
\hline & $\begin{array}{l}\text { Les autorités publiques impliquées dans un GECT vont de la commune } \\
\text { aux Etats, en passant par de nombreux autres niveaux d'organisation de } \\
\text { l'autorité publique. Le fait de pouvoir impliquer tous les niveaux de } \\
\text { pouvoir, y compris l'Etat, concernés par le développement d'un } \\
\text { Autorités publiques } \\
\text { impliquées } \\
\text { (gouvernance } \\
\text { multiniveaux) }\end{array}$ \\
$\begin{array}{l}\text { Cependant seuls } 3 \text { des } 15 \text { Etats membres abritant un GECT sont eux- } \\
\text { mêmes membres d'un GECT (BE, FR, LUX), et en réalité, seuls 6 GECT sur } \\
\text { les } 30 \text { existants peuvent être considérés comme mettant en œuvre une } \\
\text { réelle gouvernance mulitiniveaux, impliquant divers niveaux d'autorité } \\
\text { des deux côtés de la frontière. } \\
\text { En pratique, la plupart des GECT ont pour membres des autorités de } \\
\text { même niveau (communes et communes, régions et régions ...) }\end{array}$ \\
\hline
\end{tabular}

37 A ce tableau, qui se base sur des éléments factuels pour ces "clés d'entrée", il faut ajouter deux entrées plus difficiles à cerner pour appréhender le degré de construction d'un territoire transfrontalier comme projet stratégique: d'une part quels sont les acteurs initiateurs et porteurs de cette coopération; d'autre part quelles ont été les motivations pour la formalisation de la coopération sous forme de GECT.

38 Cette investigation nécessite des entretiens avec les acteurs du territoire concerné en général, et de la coopération territoriale en particulier. Dans le cadre de nos trois cas d'études approfondis (voir supra), une centaine d'acteurs ont été interrogés.

39 Les opinions des personnes interrogées quant aux forces initiatrices et mobilisatrices de la coopération montrent une convergence quant à l'importance de la volonté politique à différents niveaux territoriaux. Quels que soient les besoins fonctionnels d'un territoire, sans la volonté politique des acteurs concernés ayant autorité sur ce territoire, la coopération ne peut avancer. Cette unanimité quant à l'importance du politique ouvre cependant de nouveaux questionnements quant aux motivations de cette volonté politique, motivations qui peuvent s'avérer fort diverses.

40 Autres éléments "facilitateurs" de la coopération, les opportunités liées au cadre européen - stratégique et financier-, un cadre légal simplifié (harmonisé ?) et une évolution vers une Europe sans frontières.

41 Les motivations pour institutionnaliser ou formaliser la coopération territoriale existante varient selon le contexte; simplifier l'organisation de la gouvernance (multiniveaux et multicanaux), renforcer la possibilité d'élaborer, de présenter et de mettre en œuvre des projets, en impliquant pour ce faire tous les acteurs possibles ayant une influence sur le territoire, donner une nouvelle envergure aux territoires "périphériques", obtenir une plus grande visibilité - et lisibilité - en particulier auprès de l'UE, mais aussi auprès d'autres coopérations.

\section{Conclusions : le potentiel des GECT comme instrument d'analyse}

42 Cet article ne fait qu'effleurer les questions de la valeur ajoutée et des défis futurs de l'utilisation du règlement GECT pour la coopération territoriale en Europe ${ }^{11}$. En effet, le questionnement était ici essentiellement d'ordre méthodologique ; il s'agissait d'évaluer 
si l'approche par les GECT était prometteuse et pertinente dans le cadre d'une démarche qualitative pour évaluer le degré de construction des territoires transfrontaliers.

Les éléments analysés attestent que l'approche par les GECT est particulièrement appropriée pour une meilleure compréhension des systèmes de gouvernance, et leur éventuelle classification. Elle permet d'appréhender les acteurs multiples, les territoires à géographie variable de la coopération - $\mathrm{y}$ inclus des territoires dans des pays non membres de l'Union européenne - et l'évolution temporelle de la coopération territoriale.

Les nombreuses tentatives de "rationaliser" l'exercice des compétences publiques - outre le fait que cette apparence de rationalité peut cacher bien des motivations ambiguës bute toujours d'une part sur le fait que sans motivation - intérêt - politique, rien ne peut se faire, malgré les urgences et/ou les nécessités. D'autre part, lorsqu'une volonté politique existe vers l'instauration d'une coopération efficace, elle bute sur la difficulté de s'exercer sur un espace qui ne correspond généralement pas à une entité administrative définie, et qui, dans tous les cas, voit de multiples acteurs et autorités se partager différents enjeux et compétences (Négrier, 2005 ; Levrat et Comte, 2006). La question d'une "pertinence des périmètres" rationnelle fausse en réalité le débat: le périmètre pertinent est celui sur lequel les acteurs politiques se mettront d'accord sur base de critères fonctionnels, ou morphologiques, ou stratégiques, ou autres. Par ailleurs, ce périmètre varie selon les thématiques et au cours du temps, nécessitant un instrument de coopération pouvant assumer ces géographies variables spatialement et temporellement.

Une coopération formalisée doit donc pouvoir prendre en compte cette flexibilité, temporelle et spatiale, tout en fournissant néanmoins une structure pérenne stable sur laquelle puissent se greffer différentes évolutions. Pour cela, l'instrument GECT paraît particulièrement intéressant car, outre qu'il permet cette structure stable et cette flexibilité, il a l'avantage d'être uniforme au niveau européen : malgré les problèmes liés aux "dispositions nationales", la structure et les prérequis sont les mêmes partout en Europe. Autre avantage certain, il est un des rares - sinon unique - instrument permettant de rassembler tous les niveaux de pouvoir s'exerçant sur un territoire donné, du micro local au macro national.

46 A l'heure où l'UE réorganise sa politique de cohésion et l'ensemble de ses Fonds structurels dans une architecture unique (COM /2012/0496 final) visant une approche territoriale - intégrée de leur mise en œuvre, les GECT apparaissent également comme des outils privilégiés de la coopération territoriale. En effet, celle-ci doit pouvoir assurer de façon pérenne le développement de territoires complexes et multiples, ce qui requiert, entre autre, une sécurité juridique à long terme. Les GECT sont d'ailleurs présentés comme tels dans la proposition de la Commission (Com 2011). Leur succès, ou leur échec, sera un test quant à l'avenir de la construction d'une coopération territoriale européenne s'orientant vers une plus grande intégration européenne. Leur analyse s'avère donc aussi fructueuse à l'égard de cet enjeu européen majeur.

Enfin, les GECT sont aussi particulièrement intéressants à analyser en termes de science politique, car ils instaurent - du moins en théorie - la possibilité de nouvelles entités politiques transfrontalières "européennes" (Evrard et Chilla, 2012). Les nécessités quotidiennes des territoires transfrontaliers, et les besoins de simplification et d'harmonisation pour plus d'efficacité, sont de puissants moteurs en ce sens. A ce jour cependant les Etats membres souverains restent les maitres du jeu, comme en atteste la persistance des dispositions nationales et de la procédure d'approbation des GECT. Une 
évolution vers plus d'autonomie pour les GECT n'est pas non plus souhaitée par le Comité des Régions. ${ }^{12}$

Les enjeux de la gouvernance transfrontalière dépassent de fait le territoire transfrontalier, et interrogent en réalité la gouvernance européenne dans son ensemble. Dans le - très - lent processus de construction européenne, lié à certains abandons de souveraineté nationale vers l'Union européenne, et à une montée en puissance - encore toute relative et très différenciée selon les Etats - des niveaux d'autorité infranationale, les GECT pourraient apparaître comme des précurseurs d'une intégration européenne renforcée, impliquant tous les niveaux de pouvoirs publics, dans plusieurs États membres. Mais dans ce cas, au nom de quelle légitimité ces espaces transfrontaliers, même organisés, construits, deviendraient-ils des entités politiques d'un statut différent du reste du territoire national? A ce jour les GECT sont d'une envergure modeste, mais s'ils se développent, ils ne pourront faire l'économie d'une réflexion approfondie sur les limites et les enjeux de leur existence. En termes d'analyse, les GECT permettent dès lors une approche qui croiserait les impératifs institutionnels et souverains nationaux et les nécessités de nature économique, sociale, géographique et politique lato sensu de ces territoires infranationaux organisés, qui apparaissent comme de nouvelles entités régies par un droit européen.

\section{BIBLIOGRAPHIE}

BARCA F. (2009), An agenda for a reformed cohesion policy, A place-based approach to meeting European Union challenges and expectations, Independent Report prepared at the request of Danuta Hübner, Commissioner for Regional Policy.

CDR (2012), Avis du Comité des régions sur la révision du règlement GECT (2012/C 113/06).

CDR (2010), Avis d'initiative du Comité des régions sur les nouvelles perspectives pour la révision du règlement GECT (100/2010).

CDR (2008), Avis du Comité des régions sur Le GECT : un nouvel élan pour la coopération territoriale en Europe (308/2007).

CDR (2006), Resolution on Cooperation beyond national borders makes Europe a reality - An appeal to adopt the regulation on the European grouping on territorial cooperation (72/2006).

COM (2012) 0496 final - 2011/0276 (COD), Proposition modifiée de règlement du Parlement européen et du Conseil portant dispositions communes relatives au Fonds européen de développement régional, au Fonds social européen, au Fonds de cohésion, au Fonds européen agricole pour le développement rural et au Fonds européen pour les affaires maritimes et la pêche relevant du cadre stratégique commun, portant dispositions générales applicables au Fonds européen de développement régional, au Fonds social européen et au Fonds de cohésion, et abrogeant le règlement (CE) nº 1083/2006 du Conseil.

COM (2011) 610 final/2, 2011/0273 COD, Proposition de règlement du Parlement européen et du Conseil portant dispositions particulières relatives à la contribution du Fonds européen de développement régional à l'objectif "Coopération territoriale européenne". 
COM (2011) 610 final/2, 2011/0272 COD, Proposition de règlement du Parlement européen et du Conseil modifiant le règlement (CE) $n^{\circ}$ 1082/2006 du Parlement européen et du Conseil du 5 juillet 2006 relatif à un Groupement Européen de Coopération Territoriale (GECT) en ce qui concerne la clarification, la simplification et l'amélioration de la constitution et de la mise en cuvre de groupements de ce type.

COM (2011) 462 final, Rapport de la Commission au Parlement européen et au Conseil, "L'application du règlement (CE) n 1082/2006 relatif à un Groupement Européen de Coopération Territoriale (GECT)”.

COM (2001) 428 final, Gouvernance européenne, Un livre Blanc.

CE (2006), Règlement (ce) $n^{\circ}$ 1082/2006 du Parlement européen et du Conseil du 5 juillet 2006 relatif à un Groupement Européen de Coopération Territoriale (GECT).

DUHR S. (2012), “Mer Baltique, Danube et stratégies macro-régionales : un modèle de coopération transnationale dans l'UE ?", Notre Europe, Études et recherches, 86, septembre 2011, http:// www.notre-europe.eu/media/etud86-macro-regions-fr.pdf?pdf=ok

EVRARD E., CHILLA T. (2012), “Asymétries institutionnelles et territoriales : quelles conséquences pour la gouvernance transfrontalière ?”, in BECK J., WASSENBERG B. (dir.), Construire des ponts à travers les frontières : vers une cohésion territoriale en Europe?, Stuttgart, Steiner Verlag.

ESPON TANGO (2013), Territorial approach for new governance, Rapport final, available at www.espon.eu.

ESPON TERCO (2012), La coopération territoriale comme facteur de croissance et d'emploi, Rapport final et rapport scientifique, chapitre sur la gouvernance, disponible sur www.espon.eu.

ESPON GOVERNANCE/2 32 (2006), Governance of territorial and urban policies, disponible sur www.espon.eu.

GORZELAK G., ZAWALINSKA K. (eds.) (2013), European territories: from cooperation to integration?, Warsaw, Scholar.

HOOGHE L., MARKS G. (2001), Multilevel governance and European integration, Manham, Md, Rowman and Littlefield publishers Inc.

JOUVE B. (2003), La gouvernance urbaine en question, Paris, Elsevier.

LEVRAT N., ESPOSITO F. (éd.) (2010), Europe : de l'intégration à la Fédération, Publications de l'Institut Européen de Genève, Louvain-la-Neuve, Bruylant-Academia.

LEVRAT N., COMTE H. (éd.) (2006), Aux coutures de l'Europe. Défis et enjeux juridiques de la coopération transfrontalière, Paris, L'Harmattan.

METIS (2009, 2010, 2011), Rapport annuel sur l'évolution des GETCS, EGTC Monitoring Reports 2011, 2010, 2009 https://portal.cor.europa.eu/egtc/en-US/Publications/

METIS (2007), Groupement Européen de Coopération Territoriale (GECT) : Etat des lieux et perspectives, CDR 117/2007.

NÉGRIER E. (2005), La question métropolitaine, les politiques à l'épreuve du changement d'échelle territoriale, Grenoble, Presses universitaires de Grenoble.

\section{NOTES}

1. Le système de l'Union Européenne "est un processus évolutif, qui continue à s'inventer et à s'adapter : parfois il s'arrête, puis il se remet en mouvement. Il s'arrête parfois dans certains domaines." (Sidjanski in Levrat et Esposito, 2010, p. 111). 
2. Pour plus d'informations sur ce sujet, voir ESPON TERCO (2012) et S. Duhr (2012).

3. Le règlement est un acte normatif énoncé par l'article 288 du Traité sur le Fonctionnement de l'Union européenne (TFUE).

4. Un GECT doit se composer d'au moins deux Etats membres. Par conséquent, un GECT trilatéral (2 Etats membres +1 non membre) est possible, mais pas un bilatéral (1 Etat membre +1 non membre).

5. Une évaluation du règlement était prévue par le règlement lui même, et devait être présentée au Parlement européen et au Conseil européen en août 2011, en vue d'une révision possible de cet instrument novateur.

6. Le CDR a identifié 79 autorités, désignées par 27 états membres, qui sont habilitées à traiter les demandes des GECT, cf. 2012/C 113/06).

7. Chaque GECT implanté doit s'enregistrer auprès du Comité des Régions (CDR), en fournissant sa Convention et ses Statuts qui sont à disposition sur le site de la plateforme GECT du CDR. Le CDR publie également un rapport annuel sur l'état de mise en œuvre des GECT, Métis, 2008, 2009, 2010, 2011).

8. Les 6 GECT établis par la suite confirment les mêmes tendances.

9. Université Libre de Bruxelles/IGEAT, Université du Luxembourg, VATI (Agence hongroise de Planification territoriale, Budapest).

10. Il faut noter que les acteurs du secteur privé bénéficient depuis longtemps d'un instrument européen de même ordre, le Groupement Européen d'Intérêt Economique : Règlement (CEE) $n^{\circ}$ 2137/85 du Conseil, du 25 juillet 1985, relatif à l'institution d'un groupement européen d'intérêt économique (GEIE). Ce règlement a pour objectif de créer une nouvelle entité juridique fondée sur le droit européen, afin de faciliter et d'encourager la coopération transfrontalière.

11. Sur ce sujet, voir rapport ESPON TERCO (2012).

12. Le CDR exclut également cette évolution, cf. CDR 2012.

\section{RÉSUMÉS}

Dans le cadre d'une démarche qualitative visant à évaluer le degré de construction des territoires transfrontaliers à travers leurs systèmes de gouvernance, nous analysons ici le nouvel instrument européen GECT. Nous envisageons son potentiel et sa pertinence en tant qu'instrument d'analyse, d'une part en présentant les éléments principaux de ce règlement européen, d'autre part en proposant une grille de lecture comparative des GECT fondée sur les principales caractéristiques des systèmes de gouvernance utilisés par les GETC existants.

Within a qualitative methodologic framework aimed to evaluate the construction process of crossborder territories through their governance systems, we analyse here the new European instrument EGCT (European Grouping for Territorial Cooperation). We investigate both its potential as well as its relevance as analytical instrument. Therefore, we present the main elements of this European regulation, and propose an analytical grid for EGTCS, based on the characteristics of the governance systems used by existing EGCTs. 
INDEX

Mots-clés : gouvernance, transfrontalier, territoire, GECT, Groupement Européen de Coopération Territoriale, règlement européen

Keywords : governance, cross border, territory, European Grouping for Territorial Cooperation, European regulation

\section{AUTEUR}

\section{VALÉRIE BIOT}

Institut de Gestion de l'Environnement et d'Aménagement du Territoire, Université Libre de Bruxelles, vbiot@ulb.ac.be 